\title{
Transporte de pasajeros en el Área Metropolitana de Monterrey
}

Passenger Transport in the metropolitan area of Monterrey

Resumen

El tema del transporte urbano se ha abordado desde diferentes facetas, sin embargo, se ha puesto poca atención a las condiciones laborales del chofer y las consecuencias que ello tiene en el bienestar de cualquier sociedad. En el presente trabajo se pretende establecer la relación que existe entre las condiciones laborales de los choferes de autobuses de transporte público en el Área Metropolitana de Monterrey y la conducta que asumen estos en variables como la velocidad a la que conducen, las condiciones de sus unidades, la cantidad de pasajeros que lo abordan y el cumplimiento que tienen de las normas de tránsito. El resultado es que dependiendo de cómo sean las relaciones de precios y de competencia y empeño será el efecto que se tenga sobre la velocidad a la que conduce el chofer o su jornada laboral.

Palabras clave:

- Trasporte urbano

- Condiciones laborales

- Velocidad, Pasajeros

- Competencia, Accidentes
José Raúl Luyando Cuevas*

Guadalupe de Jesús Tapia Castro**

Celia Verónica Pérez Interian***
Abstract

The issue of urban transport has been tackled from different facets, however, it has paid little attention to the working conditions of the driver and the consequences it has on the wellbeing of any society. In the present work we are trying to establish the relationship that exist between working conditions of drivers of public transport buses in the metropolitan area of Monterrey and their behavior in variables such as the speed at which they drive, the conditions of their units, the number of passengers that board its buses and the compliance of the traffic rules. The result is that depending on how will be price relationships and ability and commitment of driver will be the effect they have on the velocity at which it is driving or their workday.

\section{Keywords: \\ - Urban Transportation \\ - Working Conditions \\ - Speed, Passengers \\ - Competition, Accidents}

JEL: J53, O18 y R41

\section{Introducción}

El objetivo principal de esta investigación es establecer la relación que existe entre las condiciones laborales de los choferes de autobuses de pasajeros en el Área Metropolitana de Monterrey y la conducta que asumen bajo éstas. Principalmente en lo referente a la velocidad a la que conducen, las condiciones de sus unidades, la cantidad de pasajeros que captan y el cumplimiento que tienen de las normas de tránsito.

\footnotetext{
* Profesor Tiempo Completo del Instituto de Investigaciones Sociales de la Universidad Autónoma de Nuevo León,jrlucu@gmail.com

** Estudiante del $7^{\circ}$ semestre de la Licenciatura en Gestión Municipal, de la Universidad Intercultural Maya de Quintana Roo,lupita_t_92@hotmail.com

***Estudiante del $7^{\circ}$ semestre de la Licenciatura en Gestión Municipal, de la Universidad Intercultural Maya de
} Quintana Roo, athena-93_15@hotmail.com . . . 
Un dato que puede reflejar las condiciones en que se encuentran las unidades, es el que ofrece la Agencia Estatal de Transporte en el año 2014. Señalan que solamente 27 unidades cumplen con las normas de edad establecidas en el reglamento y todas ellas pertenecen a las denominadas midibus panorámicas, las cuales, cuentan con aire acondicionado y tienen la cuotas más altas (entre 15 y 17 pesos por viaje). Por lo mismo, no es de extrañar la gran disconformidad de la población con el aumento de $20 \%$ al costo de transporte, estipuladas por el actual gobierno de Nuevo León a principios de este año (Gobierno de Nuevo León 2014). Pues a pesar de que el costo del pasaje es uno de los más altos en México, los usuarios se quejan de que las unidades están sucias y en mal estado (Arroyo, 2014). Incluso, en tres ocasiones alcaldes del área metropolitana de Monterrey interpusieron una controversia constitucional con el objetivo de eliminar el alza de la tarifa del transporte, pero en ninguna de ellas lograron su propósito (Informe Académico Infotrac UANL, 2014).

\begin{tabular}{|c|c|c|c|c|c|c|c|c|}
\hline & \multicolumn{4}{|c|}{ Servicio Sin Aire Acondicionado } & \multicolumn{4}{|c|}{ Servicio Con Aire Acondicionado } \\
\hline & \multicolumn{3}{|c|}{$\begin{array}{l}\text { Unidades con } \\
\text { Sistema de Prepago }\end{array}$} & \multirow{2}{*}{$\begin{array}{c}\text { Unidades } \\
\text { Sin Sistema } \\
\text { de Prepago } \\
\text { Pago en } \\
\text { Efectivo }\end{array}$} & \multicolumn{3}{|c|}{$\begin{array}{l}\text { Unidades con } \\
\text { Sistema de Prepago }\end{array}$} & \multirow{2}{*}{$\begin{array}{l}\text { Unidades } \\
\text { Sin Sistema } \\
\text { de Prepago } \\
\text { Pago en } \\
\text { Efectivo } \\
\end{array}$} \\
\hline & $\begin{array}{l}\text { Pago con } \\
\text { Tarjeta de } \\
\text { Prepago }\end{array}$ & $\begin{array}{l}\text { Pago en } \\
\text { Efectivo }\end{array}$ & $\begin{array}{l}\text { Aumento a } \\
\text { Pago con Tar- } \\
\text { jeta de Prepago } \\
\text { aplicable el día } \\
\text { último de cada } \\
\text { mes del } 2014\end{array}$ & & $\begin{array}{l}\text { Pago con } \\
\text { Tarjeta de } \\
\text { Prepago }\end{array}$ & $\begin{array}{l}\text { Pago en } \\
\text { Efectivo }\end{array}$ & $\begin{array}{c}\text { Aumento a } \\
\text { Pago con Tar- } \\
\text { jeta de Prepago } \\
\text { aplicable el día } \\
\text { último de cada } \\
\text { mes del } 2014\end{array}$ & \\
\hline & \multicolumn{8}{|c|}{ Radial y Midibús Panorámico } \\
\hline Tarifa Ordinaria & $\$ 8.71$ & $\$ 12.00$ & $\$ 0.04$ & $\$ 7.50$ & $\$ 9.99$ & $\$ 12.00$ & $\$ 0.05$ & $\$ 8.50$ \\
\hline \multirow{2}{*}{$\begin{array}{l}\text { Tarifa Preferencial } \\
\text { (estudiantes y tercera edad) }\end{array}$} & $\$ 5.39$ & $\$ 12.00$ & $\$ 0.02$ & $\$ 4.50$ & $\$ 6.67$ & $\$ 12.00$ & $\$ 0.03$ & $\$ 5.50$ \\
\hline & \multicolumn{8}{|c|}{ Periférico Panorámico } \\
\hline Tarifa Ordinaria & $\$ 9.23$ & $\$ 12.00$ & $\$ 0.04$ & $\$ 8.00$ & $\$ 10.51$ & $\$ 12.00$ & $\$ 0.05$ & $\$ 9.00$ \\
\hline \multirow{2}{*}{$\begin{array}{l}\text { Tarifa Preferencial } \\
\text { (estudiantes y tercera edad) }\end{array}$} & $\$ 5.39$ & $\$ 10.00$ & $\$ 0.02$ & $\$ 4.50$ & $\$ 6.67$ & $\$ 12.00$ & $\$ 0.03$ & $\$ 5.50$ \\
\hline & \multicolumn{8}{|c|}{ Suburbano Panorámico } \\
\hline Tarifa Ordinaria & $\$ 14.13$ & $\$ 16.00$ & $\$ 0.05$ & $\$ 12.50$ & $\$ 15.16$ & $\$ 17.00$ & $\$ 0.05$ & $\$ 13.50$ \\
\hline \multirow{2}{*}{$\begin{array}{l}\text { Tarifa Preferencial } \\
\text { (estudiantes y tercera edad) }\end{array}$} & $\$ 7.24$ & $\$ 16.00$ & $\$ 0.03$ & $\$ 6.00$ & $\$ 8.55$ & $\$ 17.00$ & $\$ 0.04$ & $\$ 7.00$ \\
\hline & \multicolumn{8}{|c|}{ Metrobús } \\
\hline Única & $\$ 9.26$ & $\$ 12.00$ & $\$ 0.04$ & $\$ 8.00$ & $\$ 10.50$ & $\$ 12.00$ & $\$ 0.05$ & $\$ 9.00$ \\
\hline
\end{tabular}


Pese a las altas tarifas que cobra, también es un transporte público peligroso, principalmente por los accidentes en que se ven envueltos y los asaltos que se llegan a cometer en ellos. De enero a junio de 2014, se han registrado un total de 109 accidentes de midibuses, donde se considera que el responsable es el conductor de la unidad (Agencia para la racionalización y modernización del transporte público de nuevo león, 2014). Estos accidentes son causados, principalmente, por la competencia por pasajeros. Lo que provoca que el conductor vaya a altas velocidades, sobrecargue el autobús de pasajeros o ponga poca atención al subir y bajar pasajeros.

\section{Gráfica I \\ Accidentes mensuales del transporte urbano como responsable en 2011-2014}

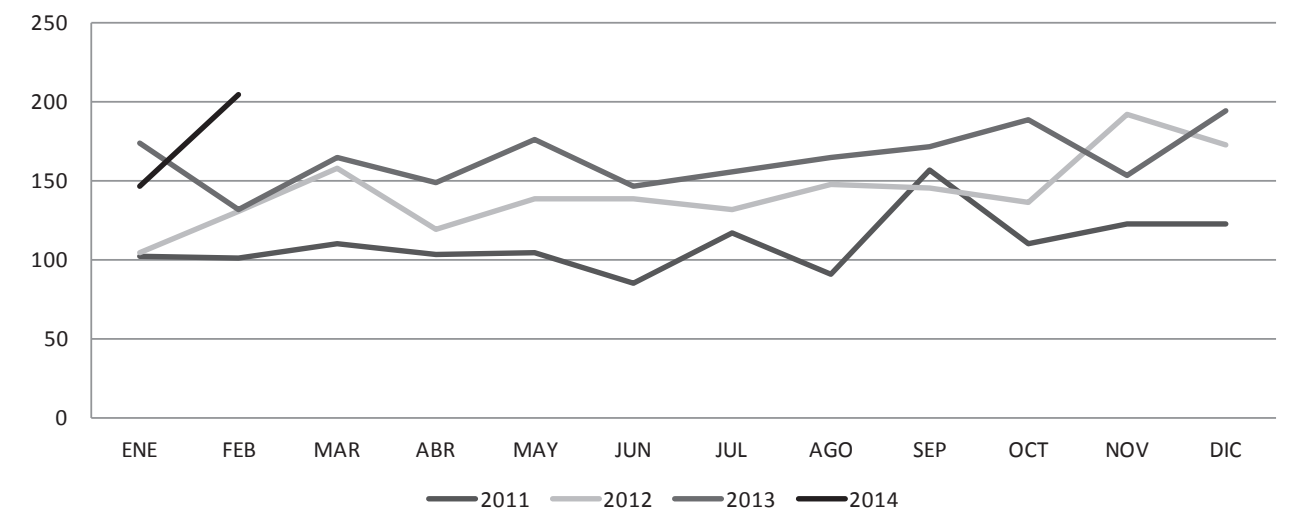

Fuente: Secretarías de Vialidad y Tránsito de los Municipios de Monterrey, San Nicolás de los Garza, Guadalupe y San Pedro, Garza García 2014.

Además de lo anterior, hay que tomar en cuenta que los costos de los insumos para este servicio han aumentado en los últimos tiempos, principalmente en lo referente a la gasolina, pero también las refacciones. Esto presiona a los dueños de las unidades a pedir mayores cuotas a los choferes para mantener sus ganancias y, por lo mismo, obligan a los mismos a una mayor competencia por el pasaje, pues es el medio como se obtiene el ingreso para pagar el salario de los choferes, las ganancias de los dueños y los costos de mantenimiento de la unidad. 
Gráfica 2

Precio combustible

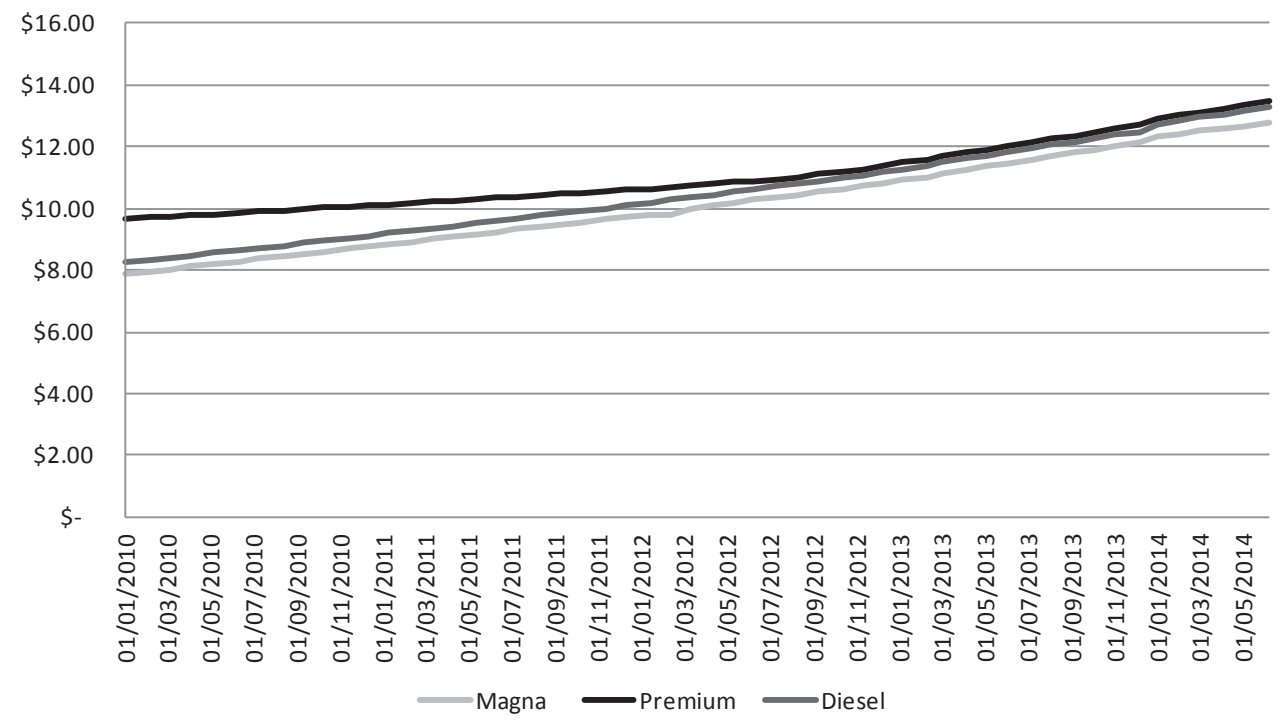

Fuente: Usuarios del transporte de carga ANTP 2014.

El Área Metropolitana de Monterrey cuenta con una población de $4.09 \mathrm{mi}-$ llones de habitantes y una extensión de $6680 \mathrm{~km}^{2}$ (Instituto Nacional de Ecología y Cambio Climático, S/F). Por su amplia extensión, las personas usuarias de este medio de transporte, se ven obligadas a desplazarse grandes distancias como parte de su rutina diaria -ya sea para llegar al trabajo, escuela u otro destino-. El desplazamiento constante de estas personas, en un espacio tan amplio como lo es la ciudad de Monterrey y su área metropolitana, provoca diversos efectos como los mencionados por autores como Fernández (1999:2):

- Congestión: aumento de tiempos de viaje de conductores, pasajeros y peatones.

- Riesgo: aumento del número y gravedad de accidentes de tránsito.

- Polución: aumento de emisiones contaminantes atmosféricos.

- Ruido: aumento del nivel de ruido y vibraciones en calles y edificios.

- Segregación del entorno: aumento en la distancia y el tiempo de cruce de cauces vehiculares.

- Intimidación: disminución del uso de las calles para otros fines (estar, jugar, pasear).

- Intrusión visual: disminución del campo visual por vehículos o infraestructura. 
Economía Informa núm. 387 julio - agosto • 2014 - " " " " " " " " " -

No obstante, cuando se habla de transporte público en una zona urbana, generalmente, el enfoque hace referencia a los problemas de rutas y su solución. Pero en la mayoría de los casos no se pone la atención debida a las condiciones laborales del chofer y las condiciones de las unidades. Sin embargo, esto debería ser diferente porque "son las personas las que realizan actividades en diferentes partes de la ciudad y requieren para ello un efectivo sistema de transporte. Por tanto, el objetivo de todo sistema de transporte urbano debe ser movilizar personas y no vehículos. Luego, lo que se debe buscar es maximizar la capacidad de transporte del sistema (número de personas que transporta por hora) y no la capacidad vial (número de vehículos que pueden circular por hora)" (Fernández, 1999:6). Pero sin olvidar que el actor principal de lo anterior es el chofer de la unidad.

\section{Planteamiento}

Se desarrolla una propuesta para analizar la relación que se mantiene en el sistema de servicio de transporte privado de pasajeros (exceptuando taxis) en el área urbana de Monterrey. En particular, en lo que se refiere a las condiciones en que laboran los choferes de esas unidades y sus consecuencias en el Área Metropolitana de Monterrey.

El precio del transporte está regulado por la autoridad Estatal. ${ }^{1}$ Hablamos del precio unitario, el que cobra el chofer de la unidad a cada pasajero. Por tanto, tenemos que el precio es $P=p$, donde $p$ sería el precio fijado por la autoridad pública. Si suponemos que el dueño de la unidad obtiene sus ganancias diarias del número de pasajeros que suban a ésta a lo largo del día. Los ingresos obtenidos mediante la venta de boletos, a lo largo del día, tendrán que servir para pagar el sueldo del chofer, el mantenimiento de la unidad, los insumos utilizados (principalmente gasolina o sustitutos) y las ganancias que requiere el dueño de la unidad. Por lo tanto, el ingreso total será igual a la cantidad de pasajeros que aborden la unidad a lo largo del día $(Q)$ por el precio del boleto $(p): I=p * Q$.

Si suponemos que las ganancias que requiere el dueño son constantes, es decir, no está dispuesto a que varíen (como si fuera un mark up sobre el precio). Se pueden ver como una cuota fija que el chofer debe obtener o pagar con base en los ingresos totales obtenidos durante su jornada de trabajo. La

1 En la Ley de Transporte para la Movilidad Sustentable del Estado de Nuevo León, se establece que la Agencia Estatal de Transporte está facultada para Autorizar las tarifas aplicables al transporte público de pasajeros (Nuevo León Unido). 
ganancia que pide el dueño deberá cubrir el seguro de la unidad, algún costo de reposición, parte del mantenimiento y los ingresos netos requeridos por el dueño. Entonces la cuota se podrá establecer como una parte fija de los ingresos totales obtenidos por el chofer o choferes en su jornada de trabajo.

Si las ganancias requeridas por el dueño las representamos como $\Pi_{d}$ y la cuota requerida por el dueño $\alpha$.

Entonces, tenemos que: $\pi_{d}=\alpha * I$ donde $0<\alpha<1$.

$\mathrm{Y}$ los ingresos obtenidos por el chofer serían: $w=I^{*}(1-\alpha)$. Como lo señalamos antes, los ingresos totales estarían en función de la cantidad de pasajeros que abordan la unidad. Pero la cantidad de pasajeros que abordan la unidad estaría restringida por la competencia de otras unidades, la jornada de trabajo, la capacidad de la unidad y el esfuerzo del chofer para acomodar gente al interior de la unidad.

En el anterior sentido, si los costos para el chofer aumentan, digamos, por el incremento en el costo de la gasolina o parte del mantenimiento que le toca hacer, dado que la ganancia es fija, el chofer se vería obligado a aumentar el número de pasajeros que abordan su unidad, lo cual, en un momento dado motivaría a los choferes a aumentar la velocidad, la jornada de trabajo o les dará incentivos para violar las normas de tránsito de transporte de pasajeros (como pueden ser: paradas no autorizadas e intentos para subir a la unidad mayor número de pasajeros del autorizado por las normas de seguridad). Causando con todo lo anterior, una serie de perjuicios a la sociedad en su conjunto.

De entrada, se debe establecer que la profesión de chofer se ha infravalorado, tomando en consideración que es una profesión que tiene una responsabilidad en vidas humanas, sin que esto se tome en cuenta, al momento de asignar una de estas unidades. Por tanto, estas personas deberían estar capacitadas para ofrecer un servicio, conscientes de la responsabilidad que tienen en sus manos y dar en este sentido seguridad a la sociedad. Para ser chofer en México no se requiere de un aprendizaje teórico-práctico. Por su bajo perfil, fue la salida de muchos desempleados y personas que iniciaban su carrera productiva. Si hay algo que se pueda llamar "costo de capacitación", queda claro que lo absorbe en su totalidad el chofer contratado -que sería el tiempo que utiliza el chofer para aprender a manejar una de estas unidades e hipotéticamente aprender el reglamento de tránsito y las normas que rigen al transporte de pasajeros-. El costo de capacitación estaría formado por el costo de aprender a manejar y el costo de aprender el reglamento de tránsito (si pagaran por ello), más el costo de oportunidad de aprender a manejar y aprender el reglamento de tránsito. 
Economía Informa núm. 387 julio - agosto • 2014 - " " " " " " " " " -

Estos costos serán variables, dependen o estarán en función de su capacidad intelectual y su habilidad, podemos decir, de su capital humano. El costo de oportunidad de aprender a manejar y aprender el reglamento estará en función del w esperado. El costo de capacitación se llevará a cabo, con el objetivo de obtener una licencia para conducir una de estas unidades. Pero si suponemos que existe corrupción en la oficina de licencias, esto provocaría que el chofer prefiera soportar el costo del soborno a realizar el costo de capacitación, pues este último sería más costoso (aprender a manejar correctamente, aprender e interpretar correctamente el reglamento de tránsito; además del costo de oportunidad de no laborar mientras se aprende el reglamento de tránsito y el funcionamiento y manejo del vehículo). En este caso, sería más redituable lo que se conoce como "learning-by-doing", es decir, aprender haciéndolo. El chofer se comportaría como un agente racional, al tratar de minimizar sus costos totales de aprendizaje en función del salario que espera obtener.

El bajo perfil escolar de los operadores de estas unidades, nos indicaría que el costo en aprendizaje sería alto y en muchos casos tardado. De esta forma, perdería por dos lados: el costo del aprendizaje y el costo de oportunidad de no laborar. Es sin duda más provechoso y más expedito, en un sistema corruptible, pagar el costo de la licencia más el soborno, que esperar, pagar y aprender. El monto del soborno dependerá de su poder de negociación y de los contactos con que cuente al interior del sistema. Los contactos, en ocasiones, son estructuras de poder que actúan al amparo de presiones políticas y la clara falta de una normatividad o supervisión rigurosa.

Las consecuencias perversas para la sociedad se podrán apreciar constantemente, choferes sin preparación requerida y sin conocimiento del reglamento de tránsito, las normas de seguridad y lo más importante, sin tener conciencia de la responsabilidad en vidas que conlleva su profesión. Esto sólo se toma en cuenta o se reclama cuando cualquiera de estas faltas implica la muerte de personas.

Bajo estas circunstancias nos encontramos con un sistema de transporte de pasajeros privado viciado: un chofer mal preparado y sin conciencia, un dueño que exige una ganancia constante y costos de operación crecientes. El operador, como sujeto racional, querrá maximizar la cantidad de pasajeros que abordan su unidad en su horario laboral establecido, sujeto a sus costos en gasolina y en depreciación o refacciones.

Como se menciona anteriormente, la cantidad de pasajeros que abordan una unidad estará en función de la velocidad a la que se conduce la unidad, la competencia de otras unidades por el pasaje, la jornada de trabajo que realice 
el chofer y del empeño que ponga el conductor ${ }^{2}$ y otras variables (que podríamos tomar como aleatorias, como podrían ser las descomposturas de no fácil remedio, los choques, atropellamientos). Por facilidad, proponemos un modelo en donde sólo se toman las variables que suponemos más influyen en los ingresos totales. Por tanto, vamos a suponer que la cantidad de pasajeros que aborda una unidad estará en función de la velocidad a la cual se conduzca la unidad, ponderada por la competencia en el mercado, por la jornada de trabajo realizada, ponderada por el esfuerzo realizado por el chofer y por el precio (que suponemos constante. Por lo tanto, se propone el siguiente modelo:

$\operatorname{Max} Q=\frac{1}{p}\left[V^{\gamma} * H^{\varepsilon}\right] \quad$ Sujeto a $C=P_{G} * V+P_{D} * H$

Donde, $Q$, representa la cantidad de pasajeros que aborda la unidad de nuestro chofer, $\gamma$ representa un factor de competencia, $V$ representa la velocidad a la que maneja nuestro chofer, ponderada por la competencia, es decir, a mayor competencia mayor velocidad $(\gamma>0)$. H representa la jornada de trabajo realizada por nuestro chofer, ponderada por el esfuerzo $(\varepsilon>0)$, es decir, el esfuerzo potenciaría la jornada de trabajo, en el sentido que el chofer estaría dispuesto esforzarse para acomodar más gente al interior de la unidad y obtener todos los usuarios posibles en ese lapso de tiempo.

La cantidad de pasajeros estará restringida por sus costos: el consumo de gasolina (el precio de la gasolina por la velocidad a que es conducida la unidad) y el precio de la depreciación o refacciones en su jornada laboral (en donde también se podrían incluir las multas o sobornos que se pagan a los policías de tránsito u otros similares).

Solucionando las condiciones de primer orden y despejando obtenemos, la velocidad optima demandada y la jornada de trabajo optima demandada.

$$
V=\frac{C^{*} \gamma}{P_{G}(\gamma+\varepsilon)} \text { y } H=\frac{C^{*} \varepsilon}{P_{D}(\gamma+\varepsilon)}
$$

Sustituyendo el costo $(\mathrm{C})$ y despejando obtenemos:

$$
V=\frac{P_{D}}{P_{G}} * \frac{\gamma}{\varepsilon} * H \quad \text { y } \quad H=\frac{P_{G}}{P_{D}} * \frac{\varepsilon}{\gamma} * V
$$

2 Hacer las paradas debidas, esperando a que toda la gente suba. Tratando de acomodar de la mejor forma a la gente dentro de la unidad, de tal manera que pueda ser ocupada por la cantidad recomendada por el fabricante y sea eficaz en la utilización de la unidad en este sentido. 
Economía Informa núm. 387 julio - agosto • 2014 " "

La velocidad óptima estará influida por la relación de precios, la relación de las ponderaciones y el horario laboral. La relación de precios parece la adecuada, si el precio de la gasolina se eleva, ceteris paribus, la velocidad disminuye. El chofer se obliga a manejar a una velocidad más moderada y constante para bajar el consumo de la gasolina y disminuir sus costos. Por otra parte, si el precio de la depreciación se eleva, ceteris paribus, el chofer tendrá incentivos para elevar la velocidad y buscar captar más pasajeros para elevar sus ingresos. En el caso de la relación de ponderaciones, no se debe olvidar que son elasticidades. En el numerador tendríamos lo que denominamos competencia, sería el cambio porcentual que se da en el número de pasajeros dado el cambio porcentual en la velocidad. Si ésta fuera elástica, ceteris paribus, el chofer tendría incentivos para aumentar más la velocidad, pues el incremento en la velocidad haría que captará muchos más pasajeros. En el caso del denominador tendríamos lo que se denominó esfuerzo del chofer, el cambio porcentual que se da en el número de pasajeros dado el cambio porcentual en el horario de trabajo. Esto captaría el empeño que pone el chofer en su jornada laboral para acomodar a los pasajeros de tal manera que siempre respete la capacidad máxima y buscar que siempre se encuentre cerca de ésta, hace las paradas debidas y respetar el reglamento de tránsito, para no tener multas o accidentes o dar sobornos y cuidando las condiciones de su unidad, para no tener desperfectos mayores o una mayor depreciación de su unidad. Si ésta fuera elástica, ceteris paribus, querría decir que su empeño está sirviendo y no necesita aumentar la velocidad, por el contrario la podrá bajar para buscar una velocidad más constante y menor gasto de gasolina, pues aumentar en una unidad su jornada laboral redundará en un aumento del número de pasajeros alto. Pero por otro lado, si es inelástica y sus esfuerzos no sirven para que un mayor número de pasajeros aborde su unidad, tendría incentivos para aumentar la jornada y aumentar la velocidad. Por último, otro elemento que afecta a la velocidad es jornada laboral, en una relación positiva. El chofer aumenta su jornada de trabajo, cuando los ingresos que obtiene en la jornada normal no cubren el ingreso que requiere y, por tanto, tendrá incentivos para aumentar la velocidad.

La velocidad óptima está influida por todos estos factores y dependiendo de cuál sea el valor de cada uno de ellos o sus cambios nos dirá la velocidad a la que circularían los choferes.

En México, el costo de la gasolina ha estado aumentando mes por mes, esto tendría que hacer que los choferes cuidaran el costo de este insumo, pero por otro lado, la competencia por el pasaje se ha incrementado y esto incentiva a los choferes a manejar más rápido buscando mayores ingresos o aumentar su 
jornada laboral, con lo que ello significa (una persona cansada y estresada por el tránsito y el roce con los pasajeros).

El análisis para la jornada óptima es similar. El precio de la depreciación tendría una relación inversa con la jornada laboral, si aumenta con el uso, ceteris paribus, el chofer querrá trabajar menos para que no ocurra tan rápido. Entre menor sea, el chofer querrá trabajar más horas, recordando que esto influye en las ganancias que el dueño de la unidad demanda, pues entre mayor sea la depreciación exigirá mayores ganancias para cubrir la reposición y viceversa. En el caso del precio de la gasolina la relación será directa, si el precio aumenta, ceteris paribus, la jornada de trabajo aumentaría para cubrir el costo mayor. En el caso de la competencia, si es elástica la jornada podrá disminuir, dado que estaría captando un mayor número de pasajeros al aumentar en una unidad la velocidad, pero si es inelástica, la jornada tendría que aumentar, pues está captando pocos pasajeros. En lo referente al esfuerzo, si es elástica le convendría trabajar una jornada mayor, pues aumentar estaría captando un mayor número de pasajeros al aumentar su jornada en una unidad. Como anteriormente pasaba, existe una relación entre la jornada laboral y la velocidad. Pero de nueva cuenta la jornada optima dependerá del costo de la gasolina, de la depreciación de la unidad, de la competencia en cantidad y no en precios y del empeño que ponga el chofer o hasta podríamos decir de su capacidad para desempeñar de la mejor manera su trabajo.

\section{Algunas conclusiones}

Aunque hay varias formas en que se paga a un chofer, en un sistema concesionado a privados, siempre se debe considerar que el ingreso de este servicio depende de la cantidad de pasajeros que aborden la unidad, dado que el precio por pasaje se mantiene fijó. Cuando la competencia no se da en precios, sino en cantidades, a factores como la velocidad, la cantidad de unidades y la jornada laboral de un chofer se les debe poner más atención. Así como a la capacitación que tiene el chofer de cada unidad: conocimientos sobre el reglamento de tránsito, la seguridad en la unidad y hacerlo consciente de que su trabajo está ligado a vidas humanas.

Cuando se toma en cuenta que los choferes de estas unidades no tienen preparación, ni se les obliga a que la tengan, se puede apreciar la laxitud de las normas que rigen esta profesión. No se toma en cuenta que esta profesión es una profesión de riesgo, no sólo para el propio chofer, sino para los pasajeros, los otros choferes (particulares y públicos) y para los peatones. Aunado a la 
competencia que los obliga el sistema, la relación se vuelve peligrosa y perversa, mayores ganancias requieren de dos factores que conllevan riesgos altos para la sociedad: mayor velocidad o mayor jornada de trabajo; dos factores de riesgo que muestran sus bajezas con ironía, la muerte de personas. Además de estas dos, se puede pensar en la inadecuada seguridad al interior de estas unidades, el esfuerzo que realizan los choferes para incluir más pasajeros, se traduce en la reducción de los asientos, con tal de aumentar la capacidad de la unidad y subir más pasajeros parados, además de la incomodidad, se vuelven otro factor de riesgo, no se siguen las reglas de seguridad estipuladas por el fabricante, la posición que guardan los pasajeros en los asientos, junto con la cantidad de pasajeros parados, son factores que propicia un mayor número de muertos o accidentados de gravedad al momento de presentarse cualquier incidente.

Cuando tomamos en cuenta estos factores, es casi obvio pensar que el transporte de pasajeros concesionado a privados no ha dado los frutos que se hubieran esperado, por el contrario encontramos choferes mal capacitados, autobuses sucios y en malas condiciones. Un servicio mal regulado por las autoridades o en complicidad con ellas: con normatividad jurídica y reglamentos de tránsito y seguridad que no sé cumplen. Lo que parece un círculo perverso, pues casi se sobrentiende que si se implementará la normatividad correctamente el costo del transporte aumentaría y se convertiría además de un problema de bienestar y seguridad social -como está sucediendo en muchos estados del país- en uno de altos costos. Sin lugar a dudas, un problema político-económico.

Aquí presentamos un modelo que trata de captar en lo posible la variables que antes hemos señalado. Es sólo una propuesta para poner énfasis en este problema y buscar soluciones, pues además de todo lo antes señalado, es un servicio que por la antigüedad de las unidades es uno de los que más contaminan el medio ambiente en México. Es un servicio que afecta el bienestar de cualquier sociedad, en general, se pensó que la concesión sería la solución, un sistema más cercano al privado. Sistemas como este se han implementado en muchos estados del país y el problema sigue siendo el mismo, inseguridad, falta de conciencia, de responsabilidad, de respeto por la vida humana. De aquí la pregunta que nos debemos hacer: ¿un servicio con estas características lo deben ofrecer los privados? 


\section{Bibliografía}

Agencia Estatal de Transporte (2014), Edad de la flota vehicular en la modalidad de MIDIBUS en el área metropolitana de Monterrey, La información fue solicitada a la Ing. Rita Bustamante, Directora de Planeación, Ingeniería y Vialidad, de la Agencia Estatal de Trasporte; y fue proporcionada vía correo electrónico.

Agencia para la racionalización y modernización del transporte público de nuevo león (2014), Accidentes de transporte urbano en tipo de servicio (midibus) donde el conductor participa como responsable en el periodo (enero-junio 2014), La información fue solicitada a la Ing. Rita Bustamante, Directora de Planeación, Ingeniería y Vialidad, de la Agencia Estatal de Trasporte; y fue proporcionada vía correo electrónico.

Arroyo, M. (2014), Padecen en NL transporte público caro y deficiente, Recuperado de http://www.jornada.unam.mx/2013/01/17/estados/030n2est. Consultado el 07/06/2014

Fernández, R. (1999), Análisis del problema del transporte urbano, Ciencia al día, Núm. 1. vol. 2. pp. 1-9.

Fuente Usuarios del transporte de carga ANTP (2014), Precio combustible, Recuperado de http://www.antp.org.mx/?page_id=230. Consultado el 29/07/14.

Gobierno de Nuevo León, (2014), Estadística de población en Nuevo León, Recuperado de http://www.nl.gob.mx/?P=nl_poblacion, Consultado el 24/07/14

Gobierno de Nuevo León (2014), Tarifas para el transporte público para el 2014, Recuperado de http://www.nl.gob.mx/?P=tarifa_2012, Consultado 25/06/14

Informe Académico Infotrac UANL (2014), Descartan dejar pelea contra alza en tarifas, Recuperado de http://go.galegroup.com/ps/i.do?id=GALE\%7CA359057125\& $\mathrm{v}=2.1 \& \mathrm{u}=\mathrm{uanl1} \& \mathrm{it}=\mathrm{r} \& \mathrm{p}=\mathrm{IFME} \& \mathrm{sw}=\mathrm{w} \& \mathrm{asid}=38720019373 \mathrm{fb} 977 \mathrm{fbd} 172 \mathrm{cfe} 1$ 6eded2, Consultado el 7/07/14.

Informe Académico Infotrac UANL (2014), Van de nuevo a Corte contra alza en tarifas, Recuperado de http://go.galegroup.com/ps/i.do?id=GALE\%7CA3582 $74605 \& \mathrm{v}=2.1 \& \mathrm{u}=\mathrm{uan} 11 \& \mathrm{it}=\mathrm{r} \& \mathrm{p}=\mathrm{IFME} \& \mathrm{sw}=\mathrm{w} \& \mathrm{asid}=925 \mathrm{f} 344 \mathrm{c} 46 \mathrm{~d} 1 \mathrm{ee} 7 \mathrm{f} 3$ da9804855687051, Consultado 4/07/14

Instituto Nacional de Ecología y Cambio Climático (S/F), Área Metropolitana de Monterrey, N.L., Recuperado de http://www2.inecc.gob.mx/publicaciones/ libros/652/monterrey.pdf, Consultado el 29/07/14

Nicholson, W. (2004), Teoría Microeconómica: principios básicos y ampliaciones, Ed. Thompson, España.

Nuevo León Unido, Gobierno para Todos (2014), Atribuciones de la Agencia, Consultado en la página: http://nl.gob.mx/?P=atrib_transporte_publico. El día 1 de julio del 2014. 